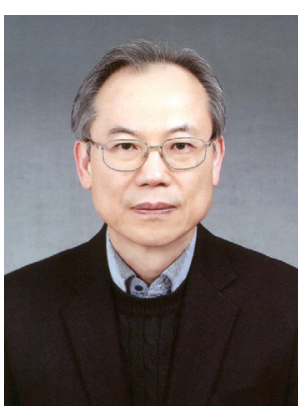

Received: July 23, 2020

Revised: August 11, 2020

Accepted: August 13, 2020

Corresponding author:

Jin Hong Chung

Department of Internal Medicine, Yeungnam University College of Medicine, 170 Hyeonchung-ro, Nam-gu, Daegu 42415, Korea

Tel: +82-53-640-6682

Fax: +82-53-620-3849

E-mail:jhchn@med.yu.ac.kr

\section{Advances in the science and treatment of respiratory diseases}

\author{
Jin Hong Chung \\ Department of Internal Medicine, Yeungnam University College of Medicine, Daegu, Korea
}

Respiratory research has developed and evolved over the past 30 years and it has been applied to clinical medicine. Significant progress has been achieved in identifying, treating, and preventing respiratory diseases. Looking back, the last 30 years revealed the following: (1) since the introduction of the flexible fiberoptic bronchoscope, bronchoscopy has been widely used as a diagnostic tool in the clinical field of respiratory medicine; (2) as bronchial asthma was identified as a chronic inflammatory airway disease, inhaled corticosteroids were approved for use as controller therapy. While most patients with asthma were treated effectively with this standard controller therapy, it was ineffective in those with severe asthma; (3) the pathogenesis and treatment of idiopathic pulmonary fibrosis (IPF) were poorly defined, and many patients with pulmonary fibrosis developed end-stage lung disease; (4) tuberculosis (TB) was expected to be eradicated by the development of powerful bactericidal drugs, such as isoniazid, rifampin, and pyrazinamide, but it has not been eradicated yet; and (5) with development in medicine and better hygiene, respiratory infections were expected to be managed and treated efficiently. However, there were concerns regarding the transmission of infections as global exchanges between countries gradually increased. This special review issue aims to highlight recent advances in the science and treatment of respiratory diseases through the articles in each of the following areas: (1) an update on the role of bronchoscopy in the diagnosis of pulmonary disease, (2) biological treatments for severe asthma, (3) therapeutic potential of targeting kinase inhibition in patients with IPF, (4) diagnosis and treatment of multidrug-resistant TB (MDR-TB), and (5) novel respiratory infectious disease in Korea.

Ahn [1] reviewed the advanced diagnostic role of bronchoscopy. Flexible bronchoscopy is an important diagnostic tool in the clinical field of respiratory medicine. Over the past decade, endobronchial ultrasound (EBUS) has been introduced in clinics. Convex probe-EBUS has been used to diagnose many central lung lesions and has replaced the surgical nodal staging of lung cancer, and radial probe-EBUS has been used to diagnose peripheral lung lesions. Recently, ultrathin bronchoscopy (UTB), electromagnetic navigational bronchoscopy (ENB), and transbronchial cryobiopsy have been introduced for the accurate diagnosis of respiratory diseases. Furthermore, flexible bronchoscopy in combination with new technologies, such as EBUS, UTB, and ENB has improved diagnostic yield.

Some patients with asthma, who used high-dose inhaled corticosteroid and long-acting beta-agonist compounds corresponding to steps 4 and 5 of the Global Initiative for Asthma guidelines, experience inadequately controlled symptoms, repeated asthma exacerbations, or sustained decline in lung function. Jin [2] reviewed the need for proper treatment of severe asthma and recently developed drugs, including biologics. These biologics reduce the symptoms of asthma, improve lung function, reduce the requirement for oral

Copyright (C) 2020 Yeungnam University College of Medicine

This is an Open Access article distributed under the terms of the Creative Commons Attribution Non-Commercial License (http://creativecommons.org/licenses/by-nc/4.0/) which permits unrestricted non-commercial use, distribution, and reproduction in any medium, provided the original work is properly cited. 
corticosteroids, and improve the quality of life of the patients. Furthermore, assessment of the patient's phenotype and endotype is essential when using these biologics. Finally, the era of personalized treatment for severe asthma has arrived.

The true nature of IPF shows that pulmonary fibrosis is a particularly critical lung disease that requires effective management. Kim et al. [3], in their continued review, evaluated the therapeutic potential of targeting kinase inhibition in patients with IPF. To develop therapeutic targets for pulmonary fibrosis, it is important to understand the key factors involved in the pathogenesis of pulmonary fibrosis and the underlying signaling pathway influencing the disease. Recently, anti-fibrotic agents, such as pirfenidone and nintedanib, have been developed and are being widely used in clinical practice for IPF management. Further intensive studies using selective kinase inhibitors should be conducted to develop therapeutic agents that delay the disease progression and improve the prognosis of IPF.

TB remains a major health hazard worldwide. MDR-TB, which shows resistance to both isoniazid and rifampicin, is one of the several barriers of TB treatment. Globally, approximately $3.4 \%$ of new TB patients and $20 \%$ of those with a previous history of TB treatment are diagnosed with MDR-TB. The treatments for MDR-TB are less effective because of highly toxic second-line drugs that require long-term use (up to 20-24 months). Jang and Chung [4] reviewed the challenges in the diagnosis and treatment of MDR-TB. The technological advance in rapid molecular drug sensitivity testing and the introduction of a new agent (bedaquiline and delamanid) and repurposed drugs (linezolid, clofazimine, and cycloserine) could improve the treatment outcomes of MDR-TB.

The current coronavirus disease 2019 (COVID-19) pandemic is potentially the biggest global health issue since the influenza pandemic of 1918. Kim [5] reviewed the new epidemic of respiratory infections. Because of the development of transportation and increased global exchanges, the transmission rate of such novel respiratory infectious diseases has increased. Since respiratory infections are not specific to a region or country, they can threaten the entire world with global trends at any time. Therefore, all nations should come together to conduct research, make efforts to prevent the spread of these diseases, and develop appropriate treatments and vaccines. From the Spanish flu to the severe acute respiratory syndrome, Middle East respiratory syndrome, and COVID-19, the unmasking of the virus responsible for these epidemics stands as a testament that worldwide medical cooperation is needed to confront emerging diseases in the future.

Finally, I hope that these articles will provide clinicians and scientists a comprehensive update on the advances in the science and treatment of respiratory diseases. Moreover, I would like to express my sincere gratitude to the editorial board of Yeungnam University Journal of Medicine for giving me the opportunity to summarize the progress of respiratory medicine. I deeply appreciate the efforts of all the authors of the articles included in this special review issue.

\section{Acknowledgments}

\section{Conflicts of interest}

No potential conflict of interest relevant to this article was reported.

\section{ORCID}

Jin Hong Chung, https://orcid.org/0000-0003-1829-3051

\section{References}

1. Ahn JH. An update on the role of bronchoscopy in the diagnosis of pulmonary disease. Yeungnam Univ J Med 2020;37:25361.

2. Jin HJ. Biological treatments for severe asthma. Yeungnam Univ J Med 2020;37:262-8.

3. Kim S, Lim JH, Woo SH. Therapeutic potential of targeting kinase inhibition in patients with idiopathic pulmonary fibrosis. Yeungnam Univ J Med 2020;37:269-76.

4. Jang JG, Chung JH. Diagnosis and treatment of multidrug-resistant tuberculosis. Yeungnam Univ J Med 2020;37:277-85.

5. Kim HJ. Novel respiratory infectious diseases in Korea. Yeungnam Univ J Med 2020;37:286-95. 